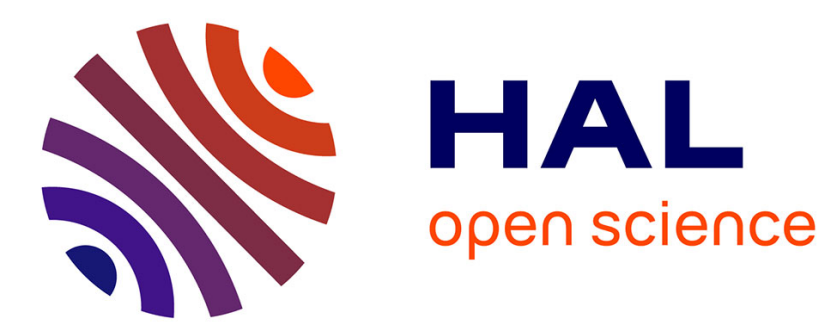

\title{
Finding solutions by finding inconsistencies
}

Ghiles Ziat, Marie Pelleau, Charlotte Truchet, Antoine Miné

\section{To cite this version:}

Ghiles Ziat, Marie Pelleau, Charlotte Truchet, Antoine Miné. Finding solutions by finding inconsistencies. CP 2018 - 24th International Conference on Principles and Practice of Constraint Programming, Aug 2018, Lille, France. pp.1-16. hal-01885769

\section{HAL Id: hal-01885769 \\ https://hal.science/hal-01885769}

Submitted on 2 Oct 2018

HAL is a multi-disciplinary open access archive for the deposit and dissemination of scientific research documents, whether they are published or not. The documents may come from teaching and research institutions in France or abroad, or from public or private research centers.
L'archive ouverte pluridisciplinaire HAL, est destinée au dépôt et à la diffusion de documents scientifiques de niveau recherche, publiés ou non, émanant des établissements d'enseignement et de recherche français ou étrangers, des laboratoires publics ou privés. 


\title{
Finding solutions by finding inconsistencies ${ }^{\star}$
}

\author{
Ghiles Ziat ${ }^{1}$, Marie Pelleau ${ }^{2}$, Charlotte Truchet ${ }^{3}$, and Antoine Miné ${ }^{1}$ \\ 1 Sorbonne Université, CNRS, Laboratoire d'Informatique de Paris 6, LIP6, F-75005 \\ Paris, France \\ 2 Université Côte d'Azur, CNRS, I3S, Nice, France \\ 3 TASC, LS2N UMR 6004, Univ. Nantes
}

\begin{abstract}
In continuous constraint programming, the solving process alternates propagation steps, which reduce the search space according to the constraints, and branching steps. In practice, the solvers spend a lot of computation time in propagation to separate feasible and infeasible parts of the search space. The constraint propagators cut the search space into two subspaces: the inconsistent one, which can be discarded, and the consistent one, which may contain solutions and where the search continues. The status of all this consistent subspace is thus indeterminate. In this article, we introduce a new step called elimination. It refines the analysis of the consistent subspace by dividing it into an indeterminate one, where the search must continue, and a satisfied one, where the constraints are always satisfied. The latter can be stored and removed from the search process. Elimination relies on the propagation of the negation of the constraints, and a new difference operator to efficiently compute the obtained set as an union of boxes, thus it uses the same representations and algorithms as those already existing in the solvers. Combined with propagation, elimination allows the solver to focus on the frontiers of the constraints, which is the core difficult part of the problem. We have implemented our method in the AbSolute solver, and present experimental results on classic benchmarks with good performances.
\end{abstract}

\section{Introduction}

Constraint solvers generally alternate two steps: propagation and exploration. The propagation step reduces the domains of the variables using the constraints. The exploration step adds hypotheses to divide the problem into several smaller sub-problems. In this article, we are interested in continuous constraint solving, where the variables have real values. In this case, the resolution of a problem usually consists in a paving of the solution space, which is not computer representable in general, using elements which are simple enough to manipulate (often floating-point boxes). This paving may correspond to an outer approximation or over-approximation of solutions, as in Ibex [6], or may correspond to an inner approximation or under-approximation as in [7].

\footnotetext{
* The work was supported, in part, by the project ANR-15-CE25-0002 Coverif from the French Agence Nationale de la Recherche, and in part by the European Research Council under Consolidator Grant Agreement 681393 - MOPSA.
} 
The efficiency of a solver depends on the choices made by the exploration process, these choices being often guided by heuristics. On discrete variables, such heuristics can for example try and provoke early failures (such as fail-first [9] or dom /w deg [4]).

On continuous variables, classic heuristics include: largest first [13], which consists in splitting the largest domain; round robin, where the domains are processed successively; or maximal smear [8], choosing the domain with the greatest slope based on the derivatives of the constraints. More recently, and closer to our work, Mind The Gaps [1] uses the idea from [8,13] and uses partial consistencies to find interesting splitting points within the domain, according to the "gaps" in the search space: splitting the domains by taking into account such gaps reduces the search space.

In this paper, we focus on covering the entire solution space of continuous problems. We propose to add a new step, complementary to constraint propagation, in the solving process: the elimination step. This step divides the search space into two sub-spaces: one containing only solutions, and the other where the constraints are indeterminate - it may contain solutions as well as nonsolutions. Our solving method alternates three steps: propagation, elimination, and exploration. It offers another way of reasoning on the constraints, since we are not only exploiting the constraints' consistencies (as does propagation) but also the constraint inconsistencies. With this improved reasoning, the interesting zones of the search space are better targeted: zones without solutions are discarded by propagation, and zones with only solutions are set aside by elimination into the solution space, which means in practice that they also are excluded from the search. The search effort can then focus on the indeterminate space - the part of the search space effectively requiring deeper exploration by the solver.

Our new step can be seen as a new contraction, in the same framework as the contractors described in [10] and used in Ibex [6] to perform a smarter exploration. We add an automatic propagation on the negation of the constraints, to identify subspaces containing only solutions. We thus reason on the negation of the constraints, hence we compute sets which are not boxes: to overcome this issue, we also add an operator on boxes to efficiently compute the difference of two boxes (or the complementary of one box in another) as a union of boxes. Thus, our method can be integrated into any solver without changing its domain representation nor modifying the propagators.

Our elimination phase relies on a notion of consistency to divide the search space and guide the search, similarly to Mind The Gaps [1] where consistency is also used to guide the search. But we go a step further by not only trying to identify the inconsistent parts of the search space (the "gaps"), but by using set complement to identify sub-spaces containing only solutions.

Our method is tailored to output an outer approximation as well as an inner approximation of the solution set: when the size of the indeterminate part is small enough and exploration stops, we can either include the indeterminate part with the definite solution space found by elimination steps to get an outer 
approximation, or return only the solution space found by elimination which is an inner approximation.

In fact, our solver can provide within the same process both an inner and an outer approximation, and due to the fact that the computed boxes better fit the constraints' shapes, this comes at no cost according to our experiments. We have tested our method on both the Coconut[16] and the MinLPLib[5] benchmarks. Our results show that first, our method computes both the inner and outer approximation with no time overhead, and second, it produces fewer boxes as an output, which makes the computed solution much more tractable.

This paper is organized as follows. Section 2 presents formally classic continuous constraint solving, on which our work is based. Section 3 introduces our new solving step: elimination. Section 4 presents experiments with our new solving method. Finally, Sect. 5 concludes and discusses future work.

\section{Preliminaries}

This section recalls basic notions of continuous Constraint Programming (CP). For a more detailed presentation, we refer the reader to [14, Chap. 16].

\subsection{Constraint Satisfaction Problems}

We consider a Constraint Satisfaction Problem (CSP) defined by: a set of $n$ variables $\mathcal{X}=\left\{x_{1}, \ldots, x_{n}\right\}$; the domain of each variable $\mathcal{D}=\left\{d_{1}, \ldots, d_{n}\right\}$, i.e., $x_{k} \in d_{k}, \forall k \in[1, n]$; and a set of $m$ constraints $\mathcal{C}=\left(C_{1}, \ldots, C_{m}\right)$. A possible assignment of the variables is a tuple in $D=d_{1} \times \cdots \times d_{n}$. A solution of the CSP is an element of $D$ satisfying all the constraints in $\mathcal{C}$. We denote as $\mathcal{S}$ the set of all solutions, i.e., $\mathcal{S}=\left\{\left(s_{1}, \ldots, s_{n}\right) \in D \mid \forall i \in\{1, \ldots, m\}, C_{i}\left(s_{1}, \ldots, s_{n}\right)\right\}$. We also denote as $\mathcal{S}_{C}$ the solution set for the constraint $C$ alone: $\mathcal{S}_{C}=\left\{\left(s_{1}, \ldots, s_{n}\right) \in\right.$ $\left.D \mid C\left(s_{1}, \ldots, s_{n}\right)\right\}$.

In the $\mathrm{CP}$ framework, variables can either be discrete or continuous. In this article, we focus on continuous, real-valued variables. Domains of variables are intervals of $\mathcal{R}$. We also assume that the bounds are (finite) floating point numbers, to be computer-representable. They can be either excluded or included. Let $\mathcal{F}$ be the set of finite floating point numbers. For $a, b \in \mathcal{F}$, we define a real-interval as the conjunction of two half-spaces $\{x \in \mathcal{R} \mid a \triangleleft x \triangleleft b\}$ where $\triangleleft \in\{<, \leq\}$, and let $\mathcal{I}$ be the set of all such intervals.

A Cartesian product of intervals is called a box. We note $\mathcal{B}=\mathcal{I}^{n}$ the set of boxes of dimension $n$. Note that our definition of interval encompasses intervals with excluded end-points which will be useful later.

For continuous CSPs, with domains in $\mathcal{I}$, the exact solution set $\mathcal{S} \subseteq \mathcal{R}^{n}$ is generally not computer-representable. Constraint solvers usually return a collection of boxes with floating-point bounds containing the solutions, the union of these being an over-approximation of $\mathcal{S}$. 


\subsection{Consistency}

The notion of local consistency is central in CP. We recall the definition of Hullconsistency [3], one of the classic local consistencies for continuous constraints.

Definition 1 (Hull-Consistency). Let $x_{1}, \ldots, x_{n}$ be variables over continuous domains represented by intervals $d_{1}, \ldots, d_{n} \in \mathcal{I}$, and $C$ a constraint. The domains are said to be Hull-consistent for $C$ if and only if $D=d_{1} \times \cdots \times d_{n}$ is the smallest floating-point box containing the solutions for $C$ in $D$.

Intuitively, no bound of a consistent box $D$ can be tightened without losing a solution of $C$. Given a constraint $C$ over domains $d_{1}, \ldots, d_{n}$, an algorithm that computes locally consistent domains $d_{1}^{\prime}, \ldots, d_{n}^{\prime}$ that contain the same solution set as $C$ in $d_{1} \times \cdots \times d_{n}$ is called a propagator for $C$. Naturally, $\forall k \in[1, n], d_{k}^{\prime} \subseteq d_{k}$. Given a constraint $C$ and domains $d_{1}, \ldots, d_{n}$, we will write $H_{C}\left(d_{1}, \ldots, d_{n}\right)$ the corresponding Hull-consistent domains and $\rho_{C}: \mathcal{B} \rightarrow \mathcal{B}$ a propagator for $C$. While we only refer to the Hull-consistency in this work, our method is based upon the propagator notion and holds for any kind of consistency.

The domains which are locally consistent for all constraints are the largest common fixpoints of all the constraint propagators $[2,15]$. In practice, propagators often compute over-approximations of the locally consistent domains. In the following, we will use the standard algorithm $\mathrm{HC} 4$ [3], which propagates continuous constraints, relying on the syntax of the constraints and interval arithmetic [11], although our method could be combined with other propagators. HC4 generally does not reach Hull consistency, in particular in case of multiple occurrences of the variables in the constraints.

Local consistency computations can be seen as deductions, performed on domains by analyzing the constraints. If the propagators return the empty set, the domains are inconsistent and the problem has no solution. Otherwise, nonempty local consistent domains are computed. This is often not sufficient to accurately approximate the solution set. In that case, choices are made on the variable values. For continuous constraints, typically a domain $d$ is chosen and split into two (or more) parts, which are in turn narrowed by the propagators. The solver alternates propagation and split phases a given precision is reached, i.e all the boxes which are still considered are smaller than a given parameter. Of course, as soon as a box is proven to contain only solutions, it can be removed from the search space and added to the solution set. Upon termination, the collection of boxes returned covers the solution set $\mathcal{S}$, under some hypotheses on the propagators and splits [2].

A solving method is said to be complete if it returns an over-approximation of the solution set (no solution is missed). It is said to be sound if it returns an under-approximation of the solution set (only solutions are returned). For problems with real variables, the solving method cannot be both complete and sound in general asbeing sound (returning only solution), requires the result to under-approximate the solution space, and being complete (returning all the solutions) requires the result to over-approximate the solution space. In practice, solving methods are often complete and not sound. 


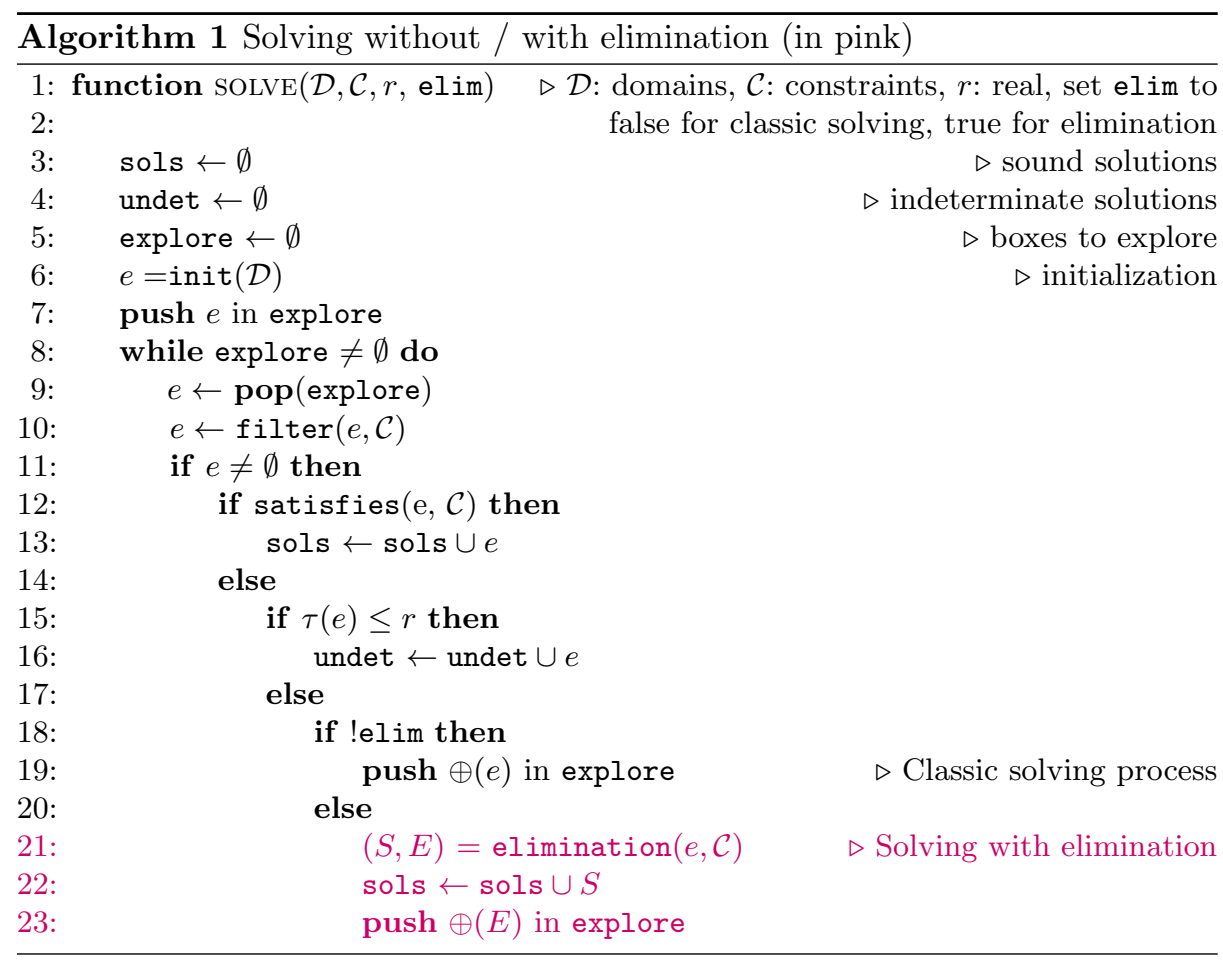

\subsection{Solving method}

In this article, we rely on the general abstract solving process described in [12], instantiated with the interval domain. The solver thus operates on boxes, as defined above. Algorithm 1 gives the pseudo-code of the abstract solving method, where $\tau \in \mathcal{B} \rightarrow \mathbb{R}$ is the precision measure and $\oplus \in \mathcal{B} \rightarrow \wp(\mathcal{B})$ is the split operator. In this section we have the elim parameter set to false, thus we do not consider the part highlighted in pink. By alternating propagation and exploration, Algorithm 1 builds a disjunction of boxes that covers the solution space. It uses three auxiliary functions: init $\in \mathcal{D} \rightarrow \mathcal{B}$, filter $\in \mathcal{B} \rightarrow \mathcal{B}$, and satisfies $\in \mathcal{B} \times \mathcal{C} \rightarrow\{$ true, false $\}$. Firstly, init creates a box from the initial domains of the problem. Then, filter corresponds to the propagation loop: it applies the propagator for each constraint in turn. Finally, satisfies checks whether a box satisfies all the constraints, that is, if it contains only solutions. This function corresponds to a contractor as defined in [6].

This solving method works as follows: at each step, the current box is tightened using the propagators on the constraints (function filter). After propagation, if the tightened box is not empty, three cases are possible:

- If the box contains only solutions (function satisfies), then it is directly added to the set of solutions sols. 


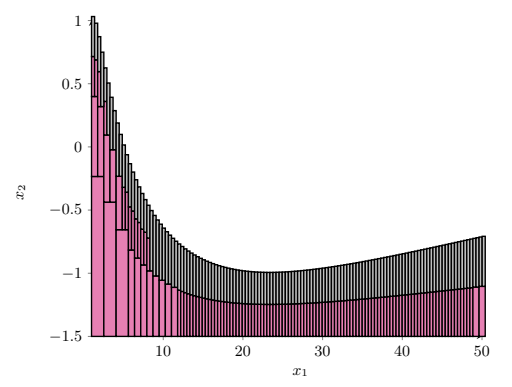

(a) Default solving

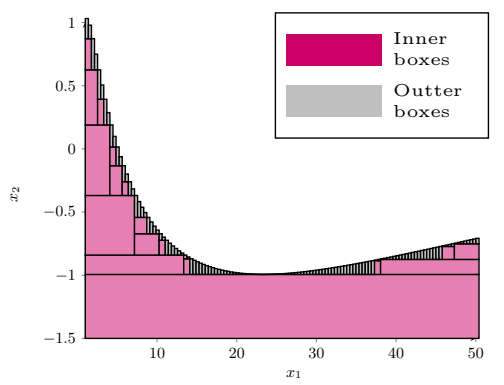

(b) Solving vith elimination

Fig. 1: (a): 127 inner boxes, 128 outer boxes. Inner boxes represent 59\% of the coverage area. Computation time 0.015s. (b): 18 inner boxes, 128 outer boxes. Inner boxes represent $92 \%$ of the coverage area. Computation time: $0.008 \mathrm{~s}$

- Otherwise, if the box is small enough with respect to a parameter $r(\tau(e) \leq$ $r)$, then it is added to the set of indeterminate solutions undet - i.e., the box, which may contain both solutions and non-solutions, is considered small enough to be left out of the search.

- Finally, if the size of the box is larger than $r$ and may contain solutions, as elim is set to false, then it is divided using a split operator $\oplus$ and the process is repeated on the resulting boxes.

Figure 1(a) shows the result obtained with Algorithm 1 with elim set to false, for a problem with two variables $x_{1} \in[1,50]$ and $x_{2} \in[-1.5,1]$ constrained by $\cos \left(\ln \left(x_{1}\right)\right)>x_{2}$. Note that this solving method can either produce an underapproximation of the solution set by considering only the inner elements, or an over-approximation by considering all the resulting elements. Figure 1(b) shows that by making different splitting choices, we could avoid computations and reach the given precision with less iterations. We achieve that using Elimination, with wich we obtain fewer, but larger inner boxes. We introduce this new step in the next section and explain how it pushes the reasoning based on the constraints one step further in order to avoid superfluous splitting steps.

\section{Elimination}

The propagation step reduces the search space by removing non-consistent subspaces. Elimination aims at reducing the search space by focusing on the frontiers of the problem. This is done in three steps: computing the elimination for each constraint, combining the result with the domains with a new difference operator, and finally integrate this mechanism in the solving process.

\subsection{Elimination for one constraint}

We introduce here the concept of elimination for a single constraint. It relies on the constraint propagator to over-approximate the set of instantiations that can 


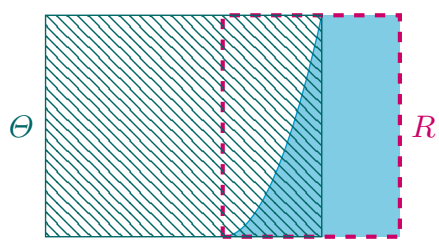

Fig. 2: Given the constraint $y \leq x^{3}$, in blue, the box $R$ over-approximates the solutions and the hatched box $\Theta$ over-approximates the inconsistencies.

not be solutions. We will refer to these instantiations as inconsistent instantiations. By elimination, the rest of the search space can only contain solutions.

In the remainder of the subsection, given a constraint $C$ and a box $D=$ $d_{1} \times \cdots \times d_{n}$, we will write $D_{C}$ the set of instantiations of $D$ that satisfy $C$ and $\overline{D_{C}}$ and, the - complementary - set of inconsistent instantiations w.r.t $C$. Thus, we have $\overline{D_{C}}=D \backslash D_{C}$. As $D_{C}, \overline{D_{C}}$ can be uncomputable, so we compute an over-approximation. For a single constraint, this can be achieved simply by reusing the propagation, over the negation of the constraint.

Definition 2. Let $x_{1}, \ldots, x_{n}$ be variables in domains $d_{1}, \ldots, d_{n}$, and $C$ a constraint on $x_{1}, \ldots, x_{n}$. We define a function $\theta_{C}: \mathcal{B} \rightarrow \mathcal{B}$ such that $\theta_{C}\left(d_{1}, \ldots, d_{n}\right)=$ $\rho_{\neg C}\left(d_{1}, \ldots, d_{n}\right)$.

Combining this function with propagation, we partition $D$ relatively to the satisfiability of $C$. Let $S_{C}=\rho_{C}\left(d_{1}, \ldots, d_{n}\right)$ and $\overline{S_{C}}=\theta_{C}\left(d_{1}, \ldots, d_{n}\right)$ be respectively the over-approximation of $D_{c}$ and $\overline{D_{C}}$, we differentiate three kinds of instantiations:

- the ones that belong to $\overline{S_{C}}$ and not to $S_{C}$, which are inconsistent,

- the ones that belong to $S_{C}$, and not to $\overline{S_{C}}$, which are consistent,

- the remaining ones that belong to both $S_{c}$ and $\overline{S_{C}}$, which are indeterminate.

Figure 2 shows an example of this partitioning. For the constraint $y \leq x^{3}$ (filled with blue), the box $\mathcal{S}_{C}$ (dashed), computed through propagation, overapproximates the solutions and the box $\Theta$ (hatched in green), computed by applying propagation over the negation of the constraint, over-approximates the inconsistencies. We can see that the complement of $\Theta$ under-approximates the set of solutions, while the complement of $R$ under-approximates the set of inconsistencies. The intersection $\Theta \cap R$ can contain both solutions and inconsistencies.

Once this partitioning is done, the inconsistent part can be discarded (as usual) and the consistent one can be directly added to the set sols of solutions. What remains is the indeterminate space in which the solving process continues. This principle is then generalized to the case of several constraints: the consistent part is the intersection of all the consistent parts associated to each constraint. Symmetrically, the inconsistent part is the union of all the inconsistent parts associated to each constraint. What remains is the indeterminate part. 
Remark In practice, in the case of continuous constraints, elimination can rely on the original propagation algorithms of the considered constraint, since we can easily compute the negation of a constraint (based on predicates $<,=, \leq$ ). It would also be valid for discrete constraints provided that the same property holds. Indeed, primitive constraints could be dealt with elimination, but handling global constraints would require to specifically define their negations and introduce dedicated propagators.

The indeterminate space is defined as an intersection of boxes, which results in a box. Hence, the solving process continues within a box, as in a classic propagation-based solver, except that the box is possibly smaller as we intersect the result of propagation with the result of elimination. However, $S_{C} \backslash \overline{S_{C}}$ is not necessarily a box. Computing this set difference requires taking the complement of a box relative to another box. In the following section, we define a set difference operator over boxes. It computes the difference as a set of boxes, that can be directly added to sols.

\subsection{Difference operator}

Given two boxes $B_{1}$ and $B_{2}$, their difference $B_{1} \backslash B_{2}$ is not necessarily a box. However, we can express it as a collection of boxes that covers $B_{1} \backslash B_{2}$. To guarantee a non-redundancy property over the result, this cover should be a partition. This would prevent boxes from overlapping and have instantiations covered by several boxes. However, a cover is sufficient to have a sound and complete resolution method, and is easier to build as we will see in the current section. Our difference operator should satisfy the following properties:

Definition 3 (Difference operator). A difference operator $\ominus: \mathcal{B} \times \mathcal{B} \rightarrow \wp(\mathcal{B})$ is a binary operator such that $\forall B_{1}, B_{2} \in \mathcal{B}$ :

(1) $\left|B_{1} \ominus B_{2}\right|$ is finite;

(2) $\forall b \in\left(B_{1} \ominus B_{2}\right) \Rightarrow b \cap B_{2}=\emptyset$;

(3) $B_{1}=\left(B_{1} \cap B_{2}\right) \cup \bigcup\left\{b \in B_{1} \ominus B_{2}\right\}$.

The first condition ensures that the solving method produces a finite set of boxes. The second one ensures that the operator eliminates from the box $B_{1}$ the values inside the box $B_{2}$. Finally, the third condition guarantees that the difference of $B_{1}$ and $B_{2}$, union $B_{2}$, covers the initial box $B_{1}$. The second condition is related to soundness and the third one to completeness.

Our difference operator on boxes works with constraints. A box can be defined as a conjunction of constraints $B=\bigwedge_{i=1, \ldots, p} c_{i}$, where each constraint $c_{i}=$ $\pm x_{i} \triangleleft a_{i}$, with $\triangleleft \in\{<, \leq\}$, gives a lower or an upper bound - not necessarily included - on $x_{i}$.

Note that it is mandatory to be able to express both strict and large inequalities. Otherwise, a problem would arise as the negation of $\pm x_{i}>a_{i}$ would not be exactly representable, and we would have no way to ensure property Def. 3.2. As the difference operator is used to compute $\mathcal{S}$, an under-approximation of the 
set of solutions, adding to $\mathcal{S}$ the closure of boxes which should actually be open, could add to it points that are not solutions to the problem, and thus break the soundness criterion.

Each $c_{i}$ defines a half-space, and the difference between a box and a halfspace is still a box. A first step is thus to compute the difference between two boxes, by considering each half space independently, as shown on Fig. 3(b).

Definition 4 (Difference for boxes). Let $B_{1}$ and $B_{2}$ be two boxes, with $B_{2}$ represented as the set of constraints $C_{2}$. The difference of $B_{1}$ and $B_{2}$ is:

$$
B_{1} \ominus B_{2} \triangleq\left\{B_{1} \cap(\neg c) \mid c \in C_{2}\right\}
$$

This naive method can result in widely overlapping boxes in the output. Nevertheless, it is an acceptable difference operator as it satisfies Def. 3:

(1) $B_{1} \ominus B_{2}$ returns a set that, associates a box to each constraint in $B_{2}$. The number of constraints in $B_{2}$ is finite, hence this set is finite (Def. 3.(1)).

(2) By definition of the intersection, the condition Def. 3.(2) is satisfied as each box in the result is included in $B_{1}$.

(3) Finally, Def. 3.(3) is also satisfied: $B_{1} \ominus B_{2}$ can be rewritten as $B_{1} \cap \overline{B_{2}}$. No solution is lost as $B_{1}$ is entirely covered by $B_{2}$ and $B_{1} \ominus B_{2}$.

Figure 3 shows an example of the application of the difference operator on two boxes. Figure $3(\mathrm{a})$ gives the initial boxes $B_{1}$ and $B_{2}$, with $B_{2}$ represented by the constraints $\left\{c_{1}, \ldots, c_{4}\right\}$. Figure $3(\mathrm{~b})$ shows the result of the naive difference operator. Here, $B_{1} \backslash B_{2}$ is covered by three elements, one per constraint of $C_{2}$, after removing the constraints that, intersected with $B_{1}$, yield the empty set $\left(c_{4}\right.$ in this case). Overlapping boxes in the output appear in a darker shades. This overlapping implies that some instantiations may be covered by more than one box: the result is redundant.

We now propose an improved difference operator in order to obtain nonoverlapping boxes when building a partition of $B_{1} \backslash B_{2}$.

Definition 5 (Non-redundant difference for boxes). Let $B_{1}$ and $B_{2}$ be two boxes and $B_{2}$ is represented by the set of constraints $C_{2}=\left\{c_{1}, \ldots, c_{p}\right\}$. The difference of $B_{1}$ and $B_{2}$ is defined as:

$$
B_{1} \ominus B_{2} \triangleq\left\{B_{1} \cap\left(\neg c_{i}\right) \cap \bigcap_{j<i} c_{j} \mid i \in\{1, \ldots, p\}\right\}
$$

For similar reasons to the naive difference operator, Def. 3.(1)-(3) is also satisfied for the non-redundant difference operator. Additionally, we strengthen the property that $B_{1} \ominus B_{2}$ is a cover for $B_{1} \backslash B_{2}$ by making this cover a partition, i.e, the elements of $B_{1} \ominus B_{2}$ are pairwise disjoints: we ensure that, for any pair of boxes $b_{i}, b_{j} \in B_{1} \ominus B_{2}$ such that $i \neq j$, we have $b_{i} \cap b_{j}=\emptyset$.

Proposition 1. $B_{1} \ominus B_{2}$ is a partition of $B_{1} \backslash B_{2}$. 


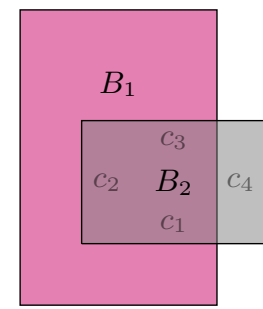

(a) Initial boxes

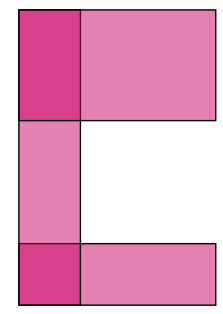

(b) Redundant difference

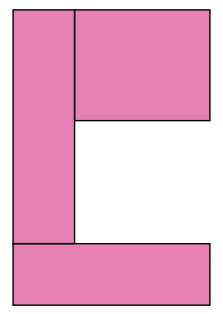

(c) Nonredundant difference

Fig. 3: Comparing naive and non-redundant difference operator: $B_{1} \ominus B_{2}$.

Proof. If $\left|B_{1} \ominus B_{2}\right|=1$ then, trivially, $B_{1} \ominus B_{2}$ is a partition of $B_{1} \backslash B_{2}$. If $\left|B_{1} \ominus B_{2}\right|>1$, we have to prove that the elements of $B_{1} \ominus B_{2}$ are pairwise disjoints. Let $C_{2}=\left\{c_{1}, \ldots, c_{p}\right\}$ be the constraints of $B_{2}$, and $b_{i}, b_{j}$ be respectively the $i$-th and the $j$-th value of $B_{1} \ominus B_{2}$ according to (2), with $i, j \in 1$..p and $i \neq j$. Then, $b_{i}$ is constrained by $\neg c_{i}$. Assuming w.l.o.g. that $i<j$, then $b_{j}$ is constrained by $c_{i}$, and $b_{i} \cap b_{j}=\emptyset$. We also have to prove that $B_{1}=B_{1} \backslash B_{2} \cup B_{2}$, or equivalently, $\cup_{i} b_{i}=B_{1} \backslash B_{2}$ : let $x \in \cup_{i} b_{i}$ be an instanciation of $B_{1}$. By definition of $B_{2}$, there is at least a constraint $c_{i} \in C_{2}$ such that $x$ does not satisfy. Let $i_{0}$ be the smallest such $i$, then $x \in b_{i_{0}}$. Thus, the whole of $B_{1} \backslash B_{2}$ is covered by the boxes $b_{i}$.

Fig. 3(c) shows the result of the non-redundant difference operator and shows that there are no overlapping darker zones (shown in a darker shade). Here, $B_{1} \backslash B_{2}$ is now partitioned into three elements, one per constraint of $C_{2}$ (once again ignoring $c_{4}$ which leads to an empty box).

\subsection{New solving step}

Computing $\widetilde{S}=\theta_{C}\left(d_{1}, \ldots, d_{n}\right) \cap \rho_{C}\left(d_{1}, \ldots, d_{n}\right)$ by employing both propagation and elimination reduces the search space, because it allows the solver to quickly identify parts of the solutions. In fact, when the propagation of $\rho_{C}$ is done, we propose an elimination step $\theta_{C}$ before splitting. Rather than performing arbitrary splits anywhere on a box, the elimination identifies parts of the box containing only solutions, and allows the solver to perform splits on the part of the search space that can not be discriminated as containing only solutions, nor as containing no solution. More precisely, elimination makes the split happen exactly at the frontier of the constraint.

Algorithm 2 gives the pseudo-code associated with the new elimination step. This algorithm processes elements that do not satisfy at least one constraint. The function complement computes $e_{n o n-c o n s}$, an over-approximation of the inconsistencies. Then, the difference operator is used to find the boxes containing only solutions. Finally, solving continues in the indeterminate search space $e \cap$ $e_{\text {non-cons }}$ (instead of e). 


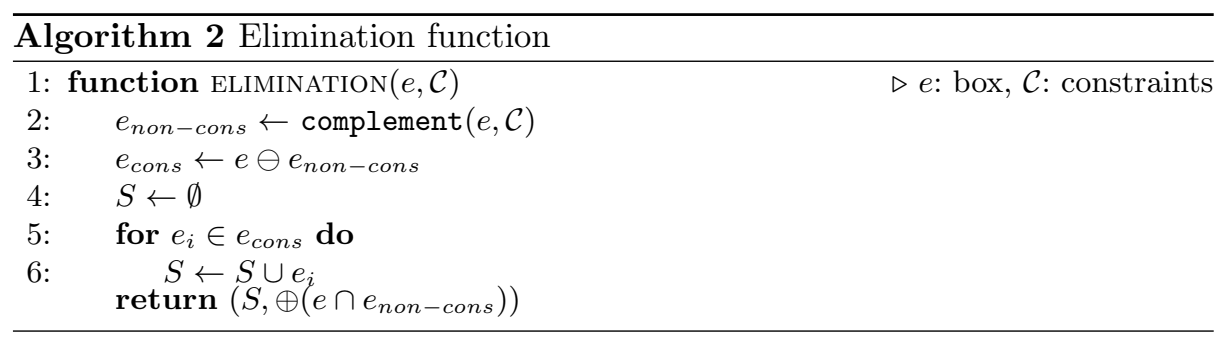

Figure 1(b) shows the results obtained with our propagation/elimination/split loop on the CSP given previously, and gives for the same precision, much more satisfactory results: we require less elements to cover more space and in a comparable amount of time, showing that this technique deduces more relevant frontier than using a simple propagation/split loop.

In the following section, we analyze the performance of our solving method.

\section{Experiments}

We have implemented our technique for boxes in the open-source solver AbSolute $^{4}$. This solver is based on the method presented in [12], where we integrated our elimination step. We rely on the abstract domain representation in AbSolute, which is based on constraints, to efficiently implement the constraint negation necessary for the elimination step. The unified constraint representation makes it possible to have a lightweight and generic difference operator.

\subsection{Protocol}

We tested our method on problems with continuous variables from the MinLPLib and the Coconut ${ }^{5}$ benchmarks. For minimization problems, we first transform them into satisfaction problems, which can be handled by the solver. This transformation consists in adding an objective variable to the problem that will act as the value to minimize. Default bounds for unconstrained variables are set to $-10^{7}$ for the lower bound and $10^{7}$ for the upper bound as our method requires the domains of the variables to be bounded. All of the runs are made with a time limit set to 300 seconds and no memory limit. Precision was fixed to $10^{-3}$ (i.e., the size limit where exploration stops), and branching depth was limited by 50 . Note that limiting depth does not break soundness nor completeness as our algorithm can be tailored to be produce either a complete or a sound output after the same run: its output can be splitted into two sets: the boxes that contain only solutions and the undetermined ones. At any point of the resolution, the union

\footnotetext{
${ }^{4}$ https://github.com/mpelleau/AbSolute

${ }^{5}$ All informations about the problems can be found at http://www.gamsworld. org/minlp/minlplib/minlpstat.htm and http://www.mat.univie.ac.at/ neum/ glopt/coconut/Benchmark/Benchmark.html
} 


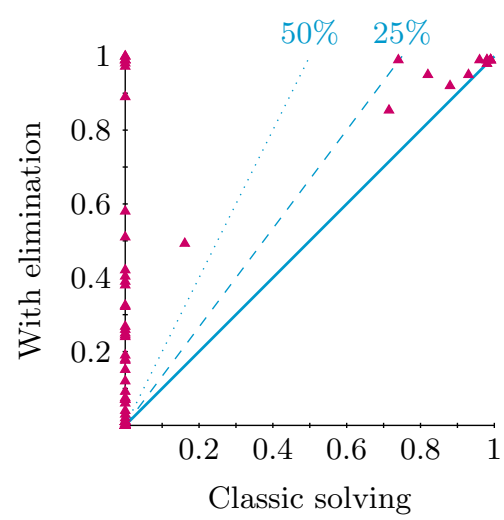

(a) Comparison of ratio

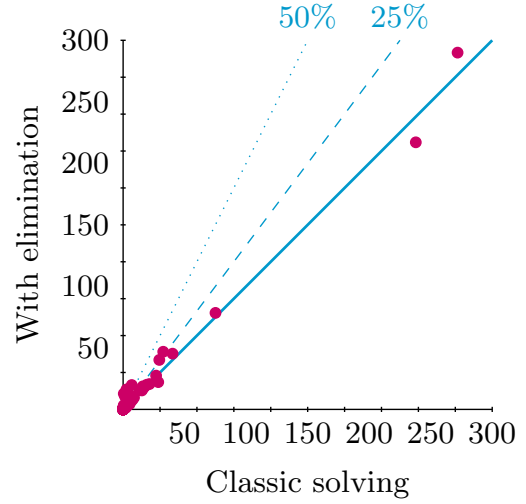

(b) Comparison of time

Fig. 4: Comparison between the classic solving method and our method. On the left, comparison of the ratio, a mark above the bisector (in plain blue) means that our method is better than the classic solving. On the right, comparison of the computation time, a mark above the bisector (in plain blue) means that our method is slower than the classic solving.

of these two sets yield a complete solution, while taking into account only the first gives a sound solution. Thus, stopping the search at a given depth, makes the resolution faster, yet less precise, but does not break neither soundness nor completeness. The solver was run on a Dell server with two 12-core Intel Xeon E5-2650 CPU at $2.20 \mathrm{GHz}$, although only one core was used, and 128GB RAM.

We have tested the solving with the elimination step against the default solving method of the AbSolute solver over all of the problems that the solver's functionalities (types, constraint, arithmetic functions) are able to cover, that is 197 problems.

\subsection{Description}

Figure 4 summarizes the results obtained with our method compared to the classic solving. Figure 4(a) compares the ratio $\delta$ of inner volume of the cover. It corresponds to $V_{i} /\left(V_{i}+V_{e}\right)$ where $V_{i}$ and $V_{e}$ are respectively the inner and outer volume. This ratio is a quality measure of the solving method: the closer this ratio is to one, the bigger is the part of the coverage that will only contain solutions. In this figure, a mark above the bisector means that our method is better than the classic one. We can see that on most of the instances, our method finds a coverage with a much smaller indeterminate space.

Figure 4(b) compares the computation time of our method to the classic one. In this figure, each mark above the bisector means that our method is slower than the classic one. As can be seen on this figure, our method is slightly slower, the elimination performing additional computation during one iteration. However, 
solving all the 197 problems took 1157 seconds with our method against 1032 seconds with the classic solving method.

\begin{tabular}{|c|c|c|c|c|c|c|c|c|c|}
\hline \multirow[b]{2}{*}{ problem } & \multirow[b]{2}{*}{$|\mathcal{X}|,|\mathcal{C}|$} & \multicolumn{4}{|c|}{ with elimination } & \multicolumn{4}{|c|}{ without elimination } \\
\hline & & $\# \mathrm{I}$ & \#E & $\delta$ & $t \mid$ & $\# \mathrm{I}$ & \#E & $\delta$ & $\mathrm{t}$ \\
\hline \multicolumn{10}{|c|}{ Coconut problems } \\
\hline abs1 & 1,2 & 2047 & 3072 & 0.99 & 0.04 & 4092 & 4096 & 0.99 & 0.06 \\
\hline aljazzaf & 2,3 & 2309 & 19405 & 0.58 & 0.89 & 0 & 14319 & 0 & 0.54 \\
\hline allinitu & 1,5 & 318 & 5066 & 0.07 & 3.26 & 0 & 5066 & 0 & 2.50 \\
\hline $\mathrm{b}$ & 4,4 & 2 & 88 & 0.39 & 0.07 & 0 & 88 & 0.00 & 0.07 \\
\hline booth & 1,2 & 90 & 45 & 0.12 & 0.11 & 0 & 45 & 0 & 0.13 \\
\hline bqp & 1,1 & 4 & 1 & 0.99 & 0.01 & 8 & 1 & 0.99 & 0.01 \\
\hline chi & 1,2 & $1.88 \mathrm{e} 6$ & $3.48 \mathrm{e} 6$ & 0.99 & 45.30 & $3.08 \mathrm{e} 6$ & $3.63 \mathrm{e} 6$ & 0.99 & 40.20 \\
\hline ex1411 & 2,5 & $1.78 \mathrm{e} 6$ & $2.59 \mathrm{e} 6$ & 0.98 & 217.08 & $1.95 \mathrm{e} 6$ & $3.74 \mathrm{e} 6$ & 0.98 & 237.89 \\
\hline ex1413 & 4,3 & 4884 & 34893 & 0.25 & \begin{tabular}{|l|}
0.52 \\
\end{tabular} & 0 & 32698 & 0 & 0.78 \\
\hline ex_newton & 2,5 & 638 & 950 & 0.95 & 0.45 & 729 & 892 & 0.93 & 0.57 \\
\hline griewank & 1,2 & 19972 & 31868 & 0.99 & 1.44 & 29645 & 35105 & 0.98 & 2.30 \\
\hline h76 & 3,4 & 24 & 174 & 0.04 & 0.05 & \begin{tabular}{|l|}
0 \\
\end{tabular} & 82 & 0 & 0.05 \\
\hline hs23 & 6,2 & 825 & 2132 & 0.99 & 0.43 & 1315 & 1801 & 0.98 & 0.58 \\
\hline kear11 & 8,8 & 0 & 844 & 0 & 0.05 & 0 & 844 & 0 & 0.05 \\
\hline ladders & 13,7 & 4 & 93 & 0.01 & 0.90 & 0 & 215 & 0.00 & 1.07 \\
\hline mickey & 2,5 & 4315 & 12709 & 0.99 & 2.40 & 8372 & 9858 & 0.99 & 2.73 \\
\hline nonlin1 & 2,3 & 1550 & 1978 & 0.95 & 0.49 & 2059 & 1772 & 0.82 & 0.69 \\
\hline nonlin2 & 3,2 & 4238 & 10560 & 0.92 & 0.39 & 8643 & 10692 & 0.88 & 0.42 \\
\hline zy2 & 3,3 & 6260 & 28147 & 0.99 & 1.00 & 13179 & 22499 & 0.74 & 0.85 \\
\hline \multicolumn{10}{|c|}{ MinLPLib problems } \\
\hline csched1a & 23,29 & 0 & 8192 & 0 & 6.44 & 0 & 8192 & 0 & 4.85 \\
\hline deb10 & 130,183 & 0 & 0 & 0 & 0.01 & 0 & 0 & 0 & 0.01 \\
\hline dosemin2d & 119,166 & 0 & 0 & 0 & 0.181 & 0 & 0 & 0 & 0.177 \\
\hline ex1222 & 4,4 & 8 & 60927 & 0.01 & 1.39 & 0 & 61787 & 0 & 0.97 \\
\hline ex1223a & 10,8 & 746 & 27097 & 0.01 & 20.60 & 0 & 48283 & 0 & 21.40 \\
\hline ex1223b & 10,8 & 820 & 44084 & 0.01 & 40.22 & 0 & 500510 & 0 & 29.41 \\
\hline gbd & 5,5 & 576 & 31829 & 0.19 & 1.27 & 0 & 22927 & 0 & 0.93 \\
\hline prob03 & 2,3 & 0 & $5.81 \mathrm{e} 6$ & 0 & 10.81 & 0 & $5.81 \mathrm{e} 6$ & 0 & 6.77 \\
\hline qapw & 256,451 & 0 & 0 & 0 & 1.20 & 0 & 0 & 0 & 1.23 \\
\hline st_e13 & 4,3 & 378 & 3102 & 0.02 & 0.05 & 0 & 18 & 0 & 0.50 \\
\hline st_miqp2 & 4,5 & 1352 & 38104 & 0.38 & 2.29 & 0 & 4564 & 0 & 0.31 \\
\hline st_miqp3 & 2,3 & 27 & 1117 & 0.24 & 0.03 & 0 & 1051 & 0 & 0.02 \\
\hline st_miqp5 & 14,8 & 187 & 2080 & 0.01 & 4.71 & 0 & 6324 & 0 & 2.38 \\
\hline st_test1 & 2,6 & 1559 & $2.44 \mathrm{e} 6$ & 0.03 & 18.70 & 0 & $2.31 \mathrm{e} 6$ & 0 & 15.97 \\
\hline st_test5 & 12,11 & 22 & 29520 & 0.01 & 7.82 & 0 & 11167 & 0.00 & 7.55 \\
\hline synthes1 & 7,7 & 97 & 33747 & 0.01 & 4.18 & 0 & 1285 & 0 & 5.16 \\
\hline tls2 & 25,38 & 0 & 18030 & 0 & 27.46 & 0 & 18030 & 0 & 26.81 \\
\hline windfac & 14,15 & 0 & 19561 & 0 & 6.66 & 0 & 19561 & 0 & 6.51 \\
\hline
\end{tabular}

Table 1: Comparing solving with and without elimination step 
For reasons of space, Table 1 highlights only some of the results representative of the behavior of our method. Those are described with respect to solving times (in seconds), cardinality of the partition and volume covered. We performed experiments on the whole benchmarks (Coconut and MinLPLib), but we do not show here the problems which time out for both methods.

The first two columns provide information about the problem: name, number of variables $|\mathcal{X}|$, and number of constraints $|\mathcal{C}|$. The rest of the table provides information on each solving methods: the number of inner (columns \#I) and outer (columns \#E) boxes.

\subsection{Analysis}

These runs highlight one very crucial feature of our method: it is able to quickly find boxes that contain only solutions of problems where the default solving method fails to do so (problems aljazzaf, allintu, ex1222, gbd, ...): on the whole benchmark, for almost $30 \%$ of the problems (58 out of 197), solving with the elimination step exhibited at least one solution while the default solving method did not succeed to do so. This comes with no time less in average: on the whole benchmark, solving with elimination was slightly slower than without (1157 seconds against 1032 seconds). In fact, $39 \%$ of the problems (39 out of 197) were solved faster with the elimination than without (problems ex1411, mickey, ex1223a, synthes1...). This illustrates the fact that results of the solver are more precise: elimination avoids unnecessary splits, better identifies the constraints' frontiers, and compute within the same process inner and outer approximations for no (or little) overhead. A deeper analysis of the results shows that the default solving method spends time splitting variables with large ranges, while elimination focuses on the shape of the constraints to locate areas than can be directly removed from the search space and added to the solution set.

Another conclusion of the analysis of this benchmark is about the solution coverage. The experiments show that the coverage of the solution space is significantly more accurate with the elimination step. On all of the runs, our method always finds a greater or equal inner volume than the one found by the default method. Moreover, it also reduces the number of elements involved in the partition in the same time, which means that the inner approximation is achieved with fewer, bigger elements. This is shown by examples chi and mickey where both methods achieve a 0.99 ratio of inner volume, only with elimination, we need half the elements required by the default solving method to do so. On the whole benchmark, on average, we need 40 times fewer elements to cover the same inner volume with elimination. This property may become very handy as it allows a better re-usability of the results since we need to treat fewer elements to cover the solution space. The $\delta$ columns indicates the part of the returned elements that corresponds to an inner approximation, i.e. contains only solutions. This ratio is always greater with the elimination step. On the whole benchmark, 
the average ratio is 0.49 of inner volume for the elimination while it is 0.27 without. This confirms that the elimination step allows the solving process to target more efficiently the parts of the search space that contain only solutions.

These good results confirm the intuition that cutting an element according to the constraints it does not satisfy can be more interesting than cutting it arbitrarily regardless of the constraints. Since solvers are often used as a precomputation for other programs, reducing the size of their output (i.e., reducing the number of boxes required to represent a solution at a given precision) can be an important feature. Also, note that, by quickly identifying solutions and removing them from the search space, the elimination step makes it possible to carry out fewer propagation and exploration steps.

\section{Conclusion}

In classic continuous constraint solvers, propagation is used to remove from the search space values that can not be solutions. We presented in this paper a new method to, symmetrically, eliminate from the search space values that can only be solutions. We have incorporated the elimination mechanism to improve the results in terms of a qualitative and quantitative criterion, also without a too large time overhead. This technique, which delays a splitting heuristic that can be inaccurate, makes it possible to take better advantage of the constraints of a problem, by reusing and adapting the same tools as propagation, combined with a difference operator we have introduced. Finally, it should be emphasized that, although it is implemented in a specific solver using abstract domains, this technique can perfectly be integrated into a more classic solver and combined with any type of propagator.

We believe that this resolution technique can be useful in many cases. For problems or zones of non-consistent instantiations forming "holes" in the solution space, or more generally, when it is non-convex, it can avoid several cutting steps by directly targeting the most relevant boundaries. This property may be particularly interesting in the context of inner-approximation applications, as shown by the experiments, or counter-example exhibition (feasibility proving) when it comes to find at least one solution as our method outperforms the default solving method in that competence.

Further research includes the development of elimination beyond boxes, for instance on polyhedra which can also be defined as a conjunction of constraints, making it possible to add a difference operator. It would also be interesting to measure the performance of this technique with other consistency and splitting heuristics.

\section{References}

1. Heikel Batnini, Claude Michel, and Michel Rueher. Mind the gaps: A new splitting strategy for consistency techniques. In Proceedings of the 11th International Conference on Principles and Practice of Constraint Programming (CP'05), volume 3709 of Lecture Notes in Computer Science, pages 77-91. Springer-Verlag, 2005. 
2. Frédéric Benhamou. Heterogeneous constraint solvings. In Proceedings of the 5th International Conference on Algebraic and Logic Programming, pages 62-76, 1996.

3. Frédéric Benhamou, Frédéric Goualard, Laurent Granvilliers, and Jean-François Puget. Revisiting hull and box consistency. In Proceedings of the 16th International Conference on Logic Programming, pages 230-244, 1999.

4. Frédéric Boussemart, Fred Hemery, Christophe Lecoutre, and Lakhdar Sais. Boosting systematic search by weighting constraints. In Proceedings of the 16th Eureopean Conference on Artificial Intelligence, (ECAI'2004), pages 146-150. IOS Press, 2004.

5. Michael R. Bussieck, Arne Stolbjerg Drud, and Alexander Meeraus. Minlplib A collection of test models for mixed-integer nonlinear programming. INFORMS Journal on Computing, 15(1):114-119, 2003.

6. Gilles Chabert and Luc Jaulin. Contractor programming. Artificial Intelligence, 173:1079-1100, 2009.

7. Hélène Collavizza, François Delobel, and Michel Rueher. Extending consistent domains of numeric CSP. In Proceedings of the 16th International Joint Conference on Artificial Intelligence, pages 406-413, 1999.

8. Eldon Hansen. Global optimization using interval analysis. Marcel Dekker, 1992.

9. Robert M. Haralick and Gordon L. Elliott. Increasing tree search efficiency for constraint satisfaction problems. In Proceedings of the 6th International Joint Conference on Artificial intelligence (IJCAI'79), pages 356-364. Morgan Kaufmann Publishers Inc., 1979.

10. Luc Jaulin and Eric Walter. Set inversion via interval analysis for nonlinear bounded-error estimation. Automatica, 29(4):1053-1064, 1993.

11. Ramon Edgar Moore. Interval Analysis. Prentice-Hall, Englewood Cliffs N. J., 1966.

12. Marie Pelleau, Antoine Miné, Charlotte Truchet, and Frédéric Benhamou. A constraint solver based on abstract domains. In Proceedings of the 14th International Conference on Verification, Model Checking, and Abstract Interpretation (VMCAI 2013), 2013.

13. Dietmar Ratz. Box-splitting strategies for the interval Gauss-Seidel step in a global optimization method. Computing, 53:337-354, 1994.

14. Francesca Rossi, Peter van Beek, and Toby Walsh, editors. Handbook of Constraint Programming. Elsevier Science Inc., New York, NY, USA, 2006.

15. Christian Schulte and Peter J. Stuckey. Efficient constraint propagation engines. Transactions on Programming Languages and Systems, 31(1):2:1-2:43, December 2008.

16. Oleg Shcherbina, Arnold Neumaier, Djamila Sam-Haroud, Xuan-Ha Vu, and TuanViet Nguyen. Benchmarking global optimization and constraint satisfaction codes. In Global Optimization and Constraint Satisfaction, First International Workshop Global Constraint Optimization and Constraint Satisfaction, COCOS 2002, Valbonne-Sophia Antipolis, France, October 2-4, 2002, Revised Selected Papers, pages 211-222, 2002. 